\title{
Research on Improved NSGA-II Algorithm and Its Application in Emergency Management
}

\author{
Xi Fang $\mathbb{D},{ }^{1,2}$ Wenwen Wang $\mathbb{D},{ }^{2}$ Lang $H e,{ }^{1,2}$ Zhangcan Huang, $^{2}$ \\ Yang Liu, ${ }^{2}$ and Liang Zhang ${ }^{2}$ \\ ${ }^{1}$ Hubei Collaborative Innovation Center for Early Warning and Emergency Response Technology, Wuhan, China \\ ${ }^{2}$ Wuhan University of Technology, Wuhan, China
}

Correspondence should be addressed to Xi Fang; fangxi@whut.edu.cn

Received 10 June 2017; Revised 15 November 2017; Accepted 13 December 2017; Published 22 January 2018

Academic Editor: Thomas Hanne

Copyright (C) 2018 Xi Fang et al. This is an open access article distributed under the Creative Commons Attribution License, which permits unrestricted use, distribution, and reproduction in any medium, provided the original work is properly cited.

\begin{abstract}
This paper constructs a dynamic multiobjective location model; three objectives are considered: the first objective maximizes the total utility of relief supplies, the second objective minimizes the number of temporary facilities needed to operate, and the third objective maximizes the satisfaction for all demand points. We propose an improved NSGA-II to solve the optimization problem. The computational experiments are divided into two sections: In the first procedure, the numerical experiment is constructed by the classical functions ZDT1, ZDT2, and DTLZ2; the results show that the proposed algorithm generates the exact Pareto front, and the convergence and uniformity of the proposed algorithm are better than the NSGA-II and MOEA/D. In the second procedure, the simulation experiment is constructed by a case in emergency management; the results show that the proposed algorithm is more reasonable than the traditional algorithms NSGA-II and MOEA/D in terms of the three objectives. It is proved that the improved NSGA-II algorithm, which is proposed in this paper, has high precision application for the sudden disaster crisis and emergency management.
\end{abstract}

\section{Introduction}

In recent years, a significant growth in casualties and economic losses caused by frequent public emergencies such as earthquakes that happened in Wenchuan killing over 67000 people, flooding in Yangtze River, and the bombing that happened in Tianjin has been seen. Emergency management has become a pressing issue in the endless emergency problems. Research on the location of relief goods distribution centers in emergency management has important application value for improving the ability of emergency management. This has prompted researchers in different fields to intensively address the problem of emergency logistics.

To provide responsive and timely service in the event of natural disasters and terrorist attacks, numerous scholars have studied emergency management and different models have been put forward; Salman and Yücel [1] considered the emergency incidents location, through maximizing cover demand which was solved by $0-1$ integer programming.
Abounacer et al. [2] took into account the minimizing distance from the distribution centers to the demand points and the noncoverage of demand points to construct multiobjective model. Since the traditional static and deterministic location models are considered as single period in which the facility location decisions and their parameters are independent of time, Ballou [3] first proposed the dynamic facility model which studies how to choose a warehouse to make the maximum profit in the planning period. Gao et al. [4] proposed a dynamic location routing problem under the factors of random and circulating flow and divided the problem into two parts constituted by a location allocation problem and a vehicles routing problem in dynamic environments. Marufuzzaman et al. [5] presented a well-known capacitated dynamic facility location problem that satisfies the customer demand at a minimum cost by determining the time period for opening, closing, or retaining an existing facility in a given location. Since the static model does not take the time factor into consideration while the dynamic model 
can consider the variable factor such as quantity demanded, energy prices, and the market growth, which becomes more adaptable and scientific in practical applications, dynamic models can be applied to multiple areas, such as combat logistics [6], electronic logistics [7], and emergency logistics [8].

Uncertainly on the timing, location, and magnitude of natural disaster, as well as how to provide timely service, impacts the disaster area posing serious challenges for disaster mitigation. Thus the emergency facility location is a multiobjective optimization problem which has to take quantities of complex factors into consideration, while the evolutionary algorithms such as GA [9], PSO [10], NSGA [11], and MOEA/D [12] are usually applied to solve such kind of problems. Gadhvi et al. [13] presented a constrained multiobjective optimization problem which is solved by NSGAII, SPEA2, and PESA-II; the results show that NSGA-II is able to yield a better Pareto front. A single algorithm usually cannot meet the demand of solving multiobjective optimization problems; then the hybrid optimization algorithm is gradually proposed. $\mathrm{Li}$ et al. [14] proposed a two-phase variable selection algorithm based on a modified NSGA-II to increase the diversity of population in the evolutionary process. Marufuzzaman et al.[5] proposed NSGA-II with chance constrained programming to solve the hybrid renewable energy system. Zade et al. [15] proposed a modified NSGAII with a new dynamic immigration operator to solve a new multiobjective hub maximal covering problem under uncertain shipments. Wang et al. [16] proposed a novel NSGA-II to solve the multiobjective optimization problem of turbine blades. Dong et al. [17] proposed an improved MOEA/D to improve global optimization capability by diversity detection and mixed population update operation. The results show that the proposed approach performs excellently compared to traditional approaches.

In this paper, we propose the dynamic multiobjective location model, and we also provide a novel solution algorithm for the stochastic model. This solution algorithm is based on a modification of elitism for NSGA-II algorithm, using a tabu search to improve the ability of local search. To the best of our knowledge, this modification is novel in the literature. We provide a computational test to show the performance of our model and to demonstrate the necessity of incorporating the modeling improvements for locating disaster response facilities. Our solution algorithm is formulated for solving the dynamic location model developed in this paper, but its basic idea should be applicable to a larger class of dynamic location problems.

The rest of the paper is organized as follows: In Section 2, we present the proposed dynamic multiobjective location model. To solve the proposed model efficiently, an improved NSGA-II approach is proposed in Section 3; Section 4 discusses the proposed algorithm's effectiveness based on the test functions ZDT1, ZDT2, and DTLZ2; Section 5 discusses the computational tests and implications arising from the study, and Section 6 summarizes the work with a discussion of the contribution of this paper, as well as the future direction.

\section{Multiobjective Optimization of Dynamic Location Model}

2.1. Problem Description. Unfortunately, natural disasters appear to be rising in scale and frequency, which may cause some buildings within the affected region to require relief goods, thus becoming a potential demand point. In a serve crisis that affects a large area, the number of demand points and the amounts of relief goods may be very large. In this case, the advance emergency centers are no longer sufficient to deal with the needs of the affected areas. To cope with these difficulties, dynamic location models needed to be constructed.

Considering that the emergencies have the nature of uncertainty and the rescue plan has the properties of being dynamic and timeliness, three objectives are considered. The first objective is to maximize the total utility of the relief goods. The second objective is to maximize the coverage rate of the needed relief goods for all demand points. The third objective is to minimize the number of temporary facilities needed to open and operate the selected distribution centers.

2.2. Parameters. The definition of parameters and decision variables used in the proposed mathematical model is summarized as follows.

Let $i$ be the fixed distribution center's number, $i=$ $1,2,3, \ldots, n ; j$ denotes the number of affected areas, $j=$ $1,2,3, \ldots, m ; s$ denotes the number of temporary distribution centers; $h_{s}$ is a two-value integer; if temporary alternative distribution centers were chosen as a resource point, $h_{s}=$ 1 ; otherwise, $h_{s}=0 ;\left(x_{i}, y_{i}\right)$ and $\left(x_{i}^{\prime}, y_{i}^{\prime}\right)$ denote the distribution's and the disaster area's coordinates; $v$ denotes the speed of truck; $f_{t}$ is a timeless function of a disaster; $x_{i j}$ denotes the demand shipped from fixed distribution center $i$ to demand point $j ; x_{s j}$ denotes the demand shipped from temporary distribution center $s$ to demand point $j$.

2.3. Mathematical Model. According to the literature [18], we know that the relationship between the time and survival of the affected people in earthquake is

$$
f(t)=e^{-0.5 *(t / 32)^{2}} .
$$

Under the particular context of providing timely service to the disaster areas, we consider the utility of relief goods as the level of timeless service. The definition of the utility of the relief goods is the total relief goods transport to the demand points within the time and survival function

$$
\begin{aligned}
& w_{k} \\
& =\sum_{i} \sum_{j} f\left(\frac{\sqrt{\left(x_{i}-x^{\prime}{ }_{j}\right)^{2}+\left(y_{i}-y^{\prime}{ }_{j}\right)^{2}}}{v_{k}}\right) \cdot x_{i j}
\end{aligned}
$$




$$
+\sum_{s} \sum_{j} h_{s} f\left(\frac{\sqrt{\left(x_{s}-x^{\prime}{ }_{j}\right)^{2}+\left(y_{s}-y_{j}^{\prime}\right)^{2}}}{v_{k}}\right) \cdot x_{s j},
$$

$\operatorname{obj} 1 f_{1}=\sum_{k} w_{k}$.

Under the context of China, we should put the fairness as one of the objectives. Our paper using the coverage rate of the needed relief goods reflects the fairness in the process of rescue; the definition is the total number of materials transported to the disaster areas divided by the amount of their needs:

$$
\begin{aligned}
& w w_{j}=\sum_{k} \sum_{i} f\left(\frac{\sqrt{\left(x_{i}-x^{\prime}{ }_{j}\right)^{2}+\left(y_{i}-y_{j}^{\prime}\right)^{2}}}{v_{k}}\right) \cdot x_{i j} \\
& +\sum_{s} \sum_{j} h_{s} f\left(\frac{\sqrt{\left(x_{s}-x^{\prime}{ }_{j}\right)^{2}+\left(y_{s}-y_{j}^{\prime}\right)^{2}}}{v_{k}}\right) \cdot x_{s j}, \\
& s f_{j}=\frac{w w_{j}}{d_{j}}, \\
& \text { obj2 } \min \left\{s f_{1}, s f_{2}, \ldots, s f_{m}\right\} \text {. }
\end{aligned}
$$

In the process of rescuing, we should also take into account the balance between the status of economic and the situation of disaster in order to avoid unnecessary waste. We use the number of temporary distribution centers reflecting the balance of emergency rescue

$$
\text { obj3 } f_{3}=\sum_{s} h_{s} .
$$

The dynamic multiobjective model can be modeled as follows:

$$
\begin{aligned}
Z=\min & \left(-f_{1},-f_{2}, f_{3}\right) \\
\text { subject to } & \sum_{j} x_{i j}=C_{i} \\
& \sum_{j} h(s) x_{s j} \leq N_{s} \\
& \sum_{i} x_{i j}+\sum_{s} h_{s} \cdot x_{s j} \leq C_{j} \\
& x_{i j}, x_{s j} \in\left\{0, R^{+}\right\}, \\
& h_{s} \in\{0,1\} .
\end{aligned}
$$

Constraint (6) ensures that when the fixed resources are exhausted, then the temporary resources can be used; constraint (7) ensures that temporary resource points that transported the number of resources in each disaster area should be less than the number of its reserves; constraint (8) ensures avoiding wasting (the number of supplies to disaster areas should be less than the demand); constraint (9) shows that the distribution of materials should be positive and $h_{i}$ is a binary value.

The demands of emergency supplies would increase gradually with the continuous development of disasters and the original material facilities could not provide sufficient supplies which may cause the situation in short supply. We put this factor into consideration to increase the robustness of the model and this is the significant difference between traditional static location model and multiobjective location model proposed by this paper. Such investigation and improvement are crucial to respond time-effectively and efficiently to the consequences of disasters and other crises where relief goods such as water, food, and first aid have to be quickly distributed in order to reduce human suffering and save lives.

\section{An Improved NSGA-II}

The problem study in this paper is a multiobjective optimization problem (MOP); the main difference between the single-objective optimization problem (SOP) and the MOP is that the solution of SOP is usually a single optimum solution corresponding to the objective function, but the MOP aims to optimize more than one objective function, thus yielding a set of solutions which are nondominated to one another in context of their corresponding objective values. To solve the MOP, the method can be divided into two ways, which are multiobjective optimal evolutionary algorithms (MOEAs) [19] and transforming the multiple objectives into single objective. However, the second method has a drawback: some solutions of Pareto front cannot be found this way which may be better than the optimal solution, so this paper chooses the MOEAs to solve the problem.

The NSGA proposed by Srinivas and Deb is an efficient sorting algorithm, but the computational complexity is $O\left(\mathrm{mN}^{3}\right)$, which is too large. In the year 1998, Deb et al. proposed a fast and elitist NSGA (NSGA-II) with the a fast nondominated sorting approach, the elitism strategy, and the crowding-distance methods which could enhance the robustness and convergence of the algorithm and the computational complexity fell to $O\left(\mathrm{mN}^{2}\right)$. But there still exist some shortcomings for NSGA-II, such as those in the iterative process; it is easy to produce duplicate individuals that cause the difficulty of searching the isolated points and with the increase of variable's dimension the convergence of traditional method is greatly reduced. It is an effective way to solve the multiobjective optimization problem by combining the global search ability of evolutionary algorithm and local search optimization method.

The modified NSGA-II is designed as a hybrid elitism for improving the local search ability in the solution space. Tabu search algorithm [20] is a generalization of the local search method that has been successfully applied to a wide class of hard optimization problems. It is a global optimization algorithm which can avoid falling into the local optimal solution by forbidding duplicate search.

In this paper the tabu search is added to the elitism strategy of NSGA-II, in order to improve the diversity and 
(1) initial: $P_{0}, F(h)=\left\{f_{1}(h), f_{2}(h), \ldots, f_{m}(h)\right\}, \forall h \in P_{0}$;

(2) $\operatorname{sort}\left(\right.$ non-dominated $\left.\left(P_{0}\right)\right)$, calculate crowding distance crowd $(h)$

(3) for $i=1$ : genmax

(4) Genetic operator: do Crossover and Mutation obtained the offsprings GAi.

(5) Tabu Search operator: $X_{0} \leftarrow$ select one individual from parent randomly which the rank $=1$;

(6) while termination not satisfied do

(7) for $j=1$ : CandidateNum

(8) $X_{j}=X_{0}+$ rand $\cdot$ scale $\cdot\left(U_{X}-L_{X}\right)$

(9) end for

(10) $N(h) \leftarrow$ non-dominate $(X) F_{k}(h)=\operatorname{opt}\{F(h), h \in N(h)\}$

(11) if $F(h) \prec F_{\mathrm{BEST}}$

(12) then $h^{*}=h, F_{\text {best }}=F\left(h^{*}\right)$

(13) else let $F_{l}(h)=\operatorname{opt}\{F(h), h \in N(h) \backslash T\}, h^{*}=h, F_{\text {best }}=F_{l}(h)$

(14) If $F_{\text {best }}$ dominates parent, add it to TS $i$

(15) update the tabu list $T, X_{0}$, then turn to step (11)

(16) end while

(17) $R_{i}=P_{o} p_{c} \cup \mathrm{GA}_{i} \cup \mathrm{TS}_{i}$

(18) sort(non-dominated $\left(R_{i}\right)$ ), calculate crowding distance $d(h)$ for each individuals in $R_{i}$;

(19) using the tournament selection select the first $P$ solutions from $R_{i}$ to $P_{i+1}$;

(20) end for

Algorithm 1: Algorithm NSGA-II-TS.

the uniformity of nondominated solutions. The combined population $R_{t}$ of the parent $P_{t}$ and the offspring including genetic operator $G_{t}$ and tabu search operator $\mathrm{TS}_{t}$ is stored for nondomination, where $t$ is the generation count. Then the nondominated set number $N$ of the combined population is obtained. This method can maintain the diversity of NSGA-II and enhance the local search ability.

Tabu search algorithm strategy is as follows.

(1) Initial Solution. The initial solution $X_{i}$ is randomly selected from the parent individuals whose rank of nondominated sorting is equal to 1 . In this case, we avoid the drawback of tabu search which is highly depending on the initial solution.

(2) Neighborhood. In the initial stage, in order to search more useful candidates, the search range of neighborhood should be large enough. With the increasing of iterations, the individual obtained is close enough to the optimal solution, so we should reduce the searching scale of neighborhood. Thus the neighborhood is produced by (10) and the scale value is updated by (11)

$$
\begin{aligned}
X_{j} & =X_{0}+\text { rand } \cdot \text { scale } \cdot\left(U_{X}-L_{X}\right), \\
\text { scale } & =\text { scale } * \lambda .
\end{aligned}
$$

(3) Candidate Solutions. For a given solution $X$, the computation is too expensive to explore its whole neighborhood $N(x)$. Therefore a candidate solutions strategy may be used for the proposed tabu search algorithm. When the next current solution $X_{\text {next }}$ is being determined, the candidate solutions of the current solution are firstly constructed, and it has been selected as the next solution. The employed candidate strategy is using nondominated sorting to rank the whole populations and calculating the crowding distance and then choosing $N$ individuals as the candidate solutions with the tournament selection.

(4) Aspiration Criterion. As an aspiration criterion, a global aspiration by objective is used. If a tabu solution $X_{t}$ has dominated the best solution $X_{\text {best }}$ obtained so far, this tabu solution $X_{t}$ is selected as the next current solution. In other words, if $X \prec X_{\text {best }}$, then $X_{t}$ will be selected as the next current solution in spite of its tabu status.

(5) Tabu List. The current individual $X$ is tabu, if and only if $\forall X_{j} \in$ Tabulist satisfies $\left|X-X_{j}\right|<\delta,\left|f_{i}\left(X_{1}\right)-f_{i}\left(X_{2}\right)\right|<\delta$.

The main steps of the algorithm are shown in Algorithm NSGA-II-TS. (see Algorithm 1).

In the search process, tabu search algorithm generated the candidate solutions in the neighborhood of its current solution, rather than being generated randomly, and it can accept suboptimal solution, which has strong climbing ability, so the probability of searching the optimal solution is relatively large. Based on the fact that importance of instability and optimization is equal, this paper proposes a hybrid evolutionary optimization algorithm by adding tabu search algorithm to the elite strategy of NSGA-II. This will on the one hand strengthen the local search ability of the solution and on the other hand retain the global search ability of NSGAII, which will increase the convergence of the global optimal solution and the accuracy of the solution.

\section{Simulations}

This section presents results from extensive computational experiments with the typical standard functions of ZDT1, ZDT2, and DTLZ2 [21] and the heuristics presented in this paper. The expression of the functions is shown in Table 1, and we want to verify the convergence and the distribution of 
TABLE 1: Test functions.

\begin{tabular}{|c|c|c|}
\hline Name & Model & Real Pareto \\
\hline ZDT1 & $\begin{array}{c}\text { Minimise } f_{1}(x)=x_{1} \text {; Minimise } f_{2}(x)=g\left(X_{M}\right)\left(1-\sqrt{\frac{f_{1}(x)}{g\left(X_{M}\right)}}\right), \\
\qquad g\left(X_{M}\right)=1+9 \sum_{x_{i} \in X_{M}} \frac{x_{i}}{n-1} \\
\text { subject } 0 \leq x_{i} \leq 1 \quad i=1,2, \ldots, n\end{array}$ & $\begin{array}{c}f_{2}=1-\sqrt{f_{1}} \\
0 \leq f_{1} \leq 1\end{array}$ \\
\hline ZDT2 & $\begin{array}{c}\text { Minimise } f_{1}(x)=x_{1} \text {; Minimise } f_{2}(x)=g\left(X_{M}\right)\left(1-\left(\frac{f_{1}(x)}{g\left(X_{M}\right)}\right)^{2}\right) \\
\qquad g\left(X_{M}\right)=1+9 \sum_{x_{i} \in X_{M}} \frac{x_{i}}{n-1} \\
\text { subject } 0 \leq x_{i} \leq 1 i=1,2, \ldots, n\end{array}$ & $\begin{aligned} f_{2} & =1-f_{1}^{2} \\
0 & \leq f_{1} \leq 1\end{aligned}$ \\
\hline DTLZ2 & $\begin{array}{c}\text { Minimize } f_{1}(X)=\left(1+g\left(X_{M}\right)\right) \cos \left(\frac{x_{1} \pi}{2}\right) \cos \left(\frac{x_{2} \pi}{2}\right) \cdots \cos \left(\frac{x_{M-2} \pi}{2}\right) \cos \left(\frac{x_{M-1} \pi}{2}\right), \\
\text { Minimize } f_{2}(X)=\left(1+g\left(X_{M}\right)\right) \cos \left(\frac{x_{1} \pi}{2}\right) \cos \left(\frac{x_{2} \pi}{2}\right) \cdots \cos \left(\frac{x_{M-2} \pi}{2}\right) \sin \left(\frac{x_{M-1} \pi}{2}\right) \\
\vdots \\
\text { Minimize } f_{M-1}(X)=\left(1+g\left(X_{M}\right)\right) \cos \left(\frac{x_{1} \pi}{2}\right) \sin \left(\frac{x_{2} \pi}{2}\right) \\
\text { Minimize } f_{M}(X)=\left(1+g\left(X_{M}\right)\right) \sin \left(\frac{x_{1} \pi}{2}\right) \\
g\left(X_{M}\right)=\sum_{x_{i} \in X_{M}}\left(x_{i}-0.5\right)^{2} \\
\text { subject } 0 \leq x_{i} \leq 1 i=1,2, \ldots, n,\end{array}$ & $\begin{array}{c}f_{1}=\cos (s) \cos (t) \\
f_{2}=\cos (s) \sin (t) \\
f_{3}=\sin (t) \\
0 \leq s, t \leq \frac{\pi}{2}\end{array}$ \\
\hline
\end{tabular}

the algorithm through the Pareto front, generation distance, and spacing.

The proposed algorithm is implemented in Intel (R) Core (TM) i5-4200H running at $3.2 \mathrm{GHz}$ with $4 \mathrm{~GB}$ RAM, and the dynamic location model is solved with Matlab R2012b (8.0.0.783). The algorithm is performed for 100 iterations with a population number of 1000 , running independently 20 times. The performance of the algorithm is analyzed in three aspects.

4.1. Pareto Front Analysis. The final Pareto sets of performing optimization process using the proposed NSGA-II-TS algorithm on the site under study, NSGA-II, and MOEA/D are compared; Figure 1 presents the Pareto sets obtained by NSGA-II-TS, NSGA-II, and MOEA/D in the case of the number of independent variables being 30 and 100, respectively.

Figure 1 presents a comparison between the real Pareto front and the final Pareto front of performing NSGA-II-TS, NSGA-II, and MOEA/D. It can be observed that the NSGAII with tabu search produces more conservative results as compared to the other algorithm. In addition, the Pareto fronts obtained from 2-objective optimization studies of ZDT1 and ZDT2 by NAGA-II basically coincide with the real Pareto front, while being superimposed over the respective planes of the other two algorithms. As the number of decision variables increases to 100 , the efficiency of the MOEA/D algorithm is significant reduced, and the approximation effect is particularly poor and needs at least 300 iterations to reach the better Pareto front. The NSGA-II algorithm can approximate the real Pareto in 100 iterations, but its effect is far less than NSGA-II-TS, which shows that, whether in small or large decision variables, NSGA-II-TS can be a better approach to the optimal Pareto front.

Similarly, Pareto fronts of 3-objective optimization studies of DTLZ2 by NSGA-II-TS, NSGA-II, and MOEA/D are presented in Figure 2. As can be seen from Figures 2(a), 2(b) and 2(c) for 3-objective optimization study, NAGA-II has a wide spread of Pareto optimal set of solutions as compared to the NSGA-II and MOEA/D. The reason behind this can be attributed to the elitism added tabu search and diversity preserving mechanism of the NSGA-II-TS. It can be observed from Figures 2(d), 2(e), and 2(f) that when the number of variables increases to 100 , the diversity Pareto set of NSGA-II and MOEA/D is significantly poor, but NSGA-II-TS can still have better converge to the real Pareto front, and the Pareto set remains diverse.

From the analysis of Pareto front, it can be concluded that, in dealing with multiobjective optimization of large-scale variables, the convergence and distribution of the proposed NSGA-II-TS algorithm have been significantly improved. In order to be more objective when describing the performance of the algorithm, this paper will illustrate the effectiveness of the algorithm by generation distance and spacing in the following section.

4.2. Convergence Analysis. Generational distance [22] is an indicator of the convergence of the nondominated set proposed by Deb et al., which shows the degree of approximation of the nondominated set to the Pareto front

$$
\operatorname{gd}=\frac{\sum_{u \in P} d\left(u, P^{*}\right)}{|P|},
$$



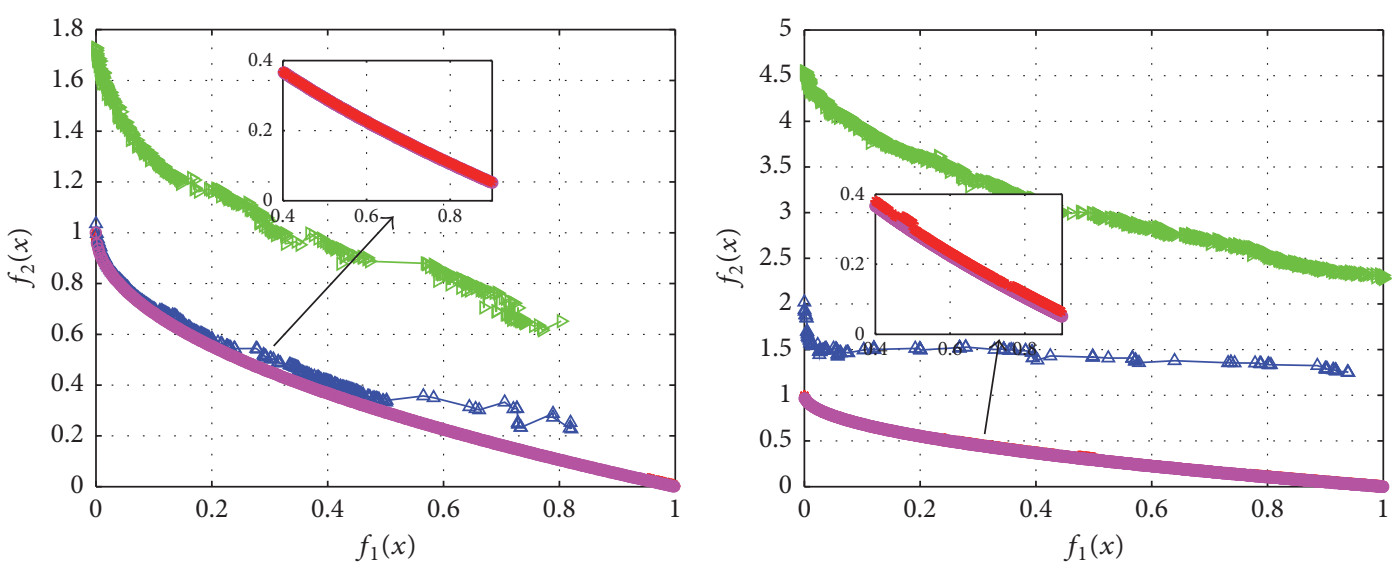

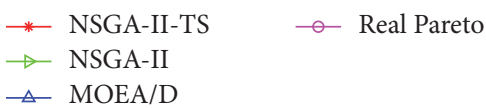

(a) ZDT1 (variables 30)

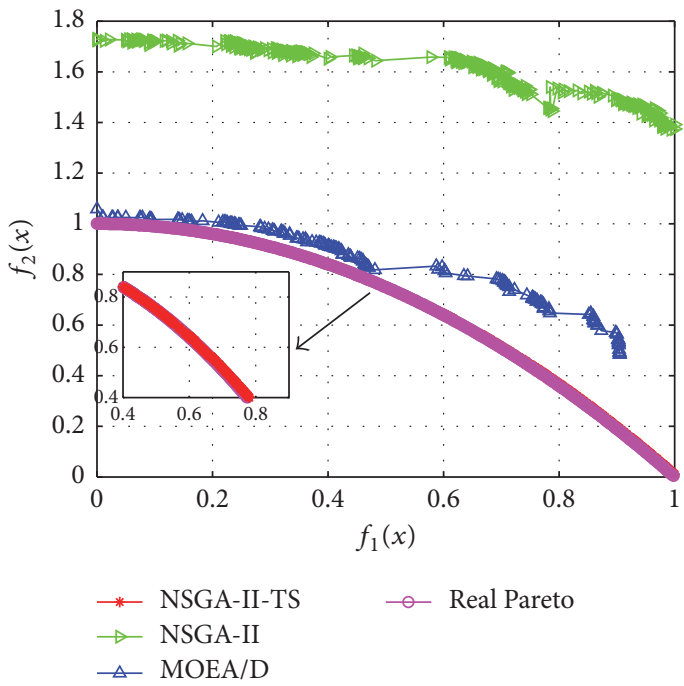

(c) ZDT2 (variables 30)

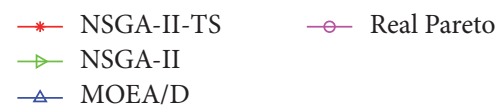

(b) ZDT1 (variables 100)

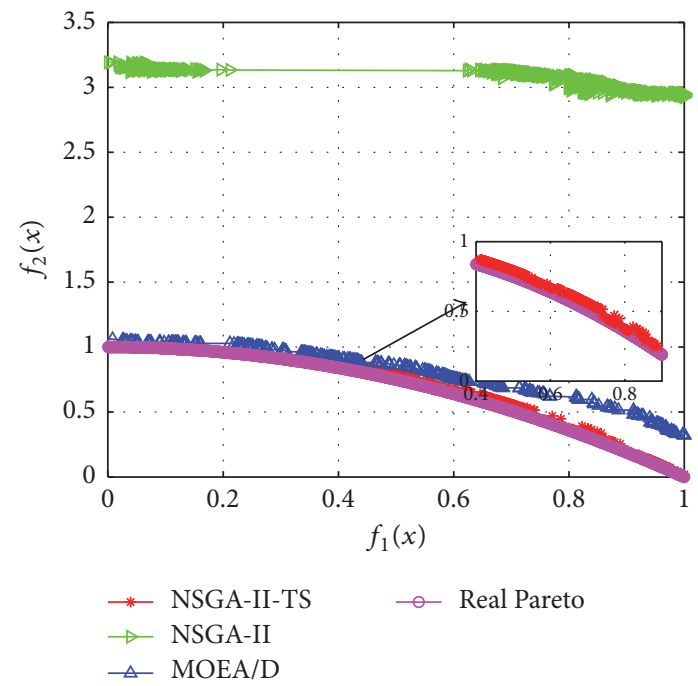

(d) ZDT2 (variables 100)

FIGURE 1: Pareto front for ZDT1 and ZDT2.

where $P^{*}$ is the sample point of uniform distribution on the optimal PF, $P$ is the nondominated set obtained by algorithm, and $d\left(u, P^{*}\right)$ is the minimum distance from points $u \in P$ to $P^{*}$.

Figure 3 shows the box plot of GD in the case of ZDT1, ZDT2, and DTLZ2 with different variables, which illustrates the dispersion of GD. Compared with NSGAII and MOEA/D, the deviation of NSGA-II-TS is smallest whenever the variables are 30 or 100, and both of NSGAII and MOEA/D have outliers, which impacts the reliability of the measures. From Figures 3(a) and 3(d), it can be seen that, with the number of independent variables increasing, the GD of NSGA-II and MOEA/D has obviously increased, but the GD of NSGA-II-TS remains small, which indicates that the convergence of NSGA-II-TS is better than the other two methods.

Table 2 shows the mean, standard deviation, and median value of the generation distance for NSGA-II-TS, NSGA-II, and MOEA/D. From Table 2, when the number of variables in ZDT1 is 30, the average generation distance of NSGA-IITS is 0.0523 , deviation is 0.1484 , and the median is 0.0030 , and in NSGA-II with the same function and variables, the mean generation distance is 0.4913 , the deviation is 0.4913 , the median is 0.5003 , the mean value of MOEA/D is 0.0672 , the deviation is 0.0154 , and the median is 0.0661 . The results show that the convergence of NSGA-II-TS is better than that of NSGA-II and MOEA/D algorithm, and the variation of each experiment is small. This property increases with the number of independent variables; when the number of independent variables increased to 100, NSGA-II-TS's generation distance value increased by only 0.005 , while the NSGA-II increased by 1.6 and MOEA/D increased by 0.21 , which indicates that the NSGA-II-TS is more stable and efficient than NSGA-II and MOEA/D in dealing with large-scale variables.

4.3. Distribution Analysis. Spacing is an index proposed by Schott [23] to evaluate the distribution of the solution, which can be used to evaluate the distributions of any two solutions 
TABLE 2: Generation distance.

\begin{tabular}{|c|c|c|c|c|c|c|c|c|c|}
\hline & \multicolumn{3}{|c|}{ NSGA-II-TS } & \multicolumn{3}{|c|}{ NSGA-II } & \multicolumn{3}{|c|}{ MOEA/D } \\
\hline & Mean & Std. dev. & Median & Mean & Std. dev. & Median & Mean & Std. dev. & Median \\
\hline \multicolumn{10}{|l|}{ ZDT1 } \\
\hline 30 & 0.0523 & 0.1484 & 0.0030 & 0.4913 & 0.0762 & 0.5003 & 0.0672 & 0.0154 & 0.0661 \\
\hline 100 & 0.10373 & 0.0096 & 0.011 & 2.0805 & 0.0550 & 2.0507 & 0.4286 & 0.3291 & 0.2253 \\
\hline \multicolumn{10}{|l|}{ ZDT2 } \\
\hline 30 & 0.0030 & 0.0012 & 0.0024 & 0.8812 & 0.0747 & 0.8588 & 0.1228 & 0.0911 & 0.1173 \\
\hline 100 & 0.0132 & 0.0097 & 0.0134 & 3.1470 & 0.7632 & 3.4995 & 0.2489 & 0.051 & 0.2634 \\
\hline \multicolumn{10}{|c|}{ DTLZ2 } \\
\hline 12 & 0.1216 & 0.0079 & 0.1227 & 0.1322 & 0.0068 & 0.1302 & 0.1863 & 0.0039 & 0.1859 \\
\hline 100 & 0.1326 & 0.0082 & 0.1327 & 0.4233 & 0.0171 & 0.417 & 0.2298 & 0.0083 & 0.2278 \\
\hline
\end{tabular}

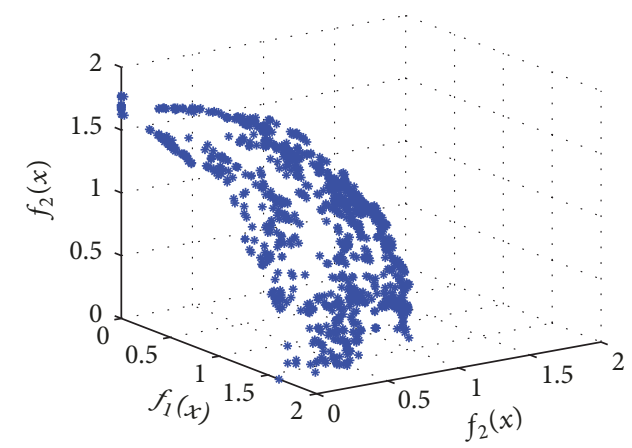

(a) NSGA-II (30 variables)

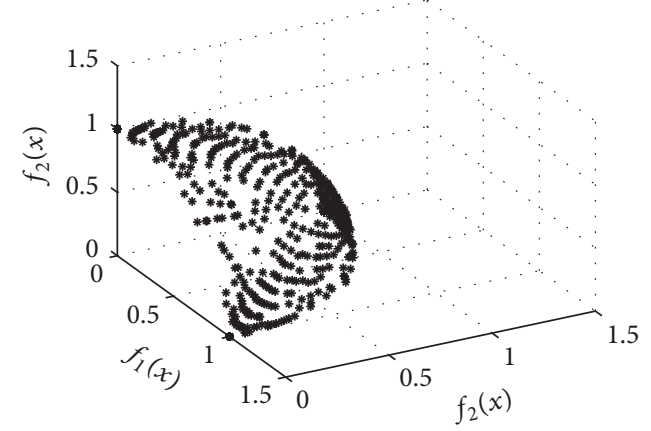

(c) MOEA/D (30 variables)

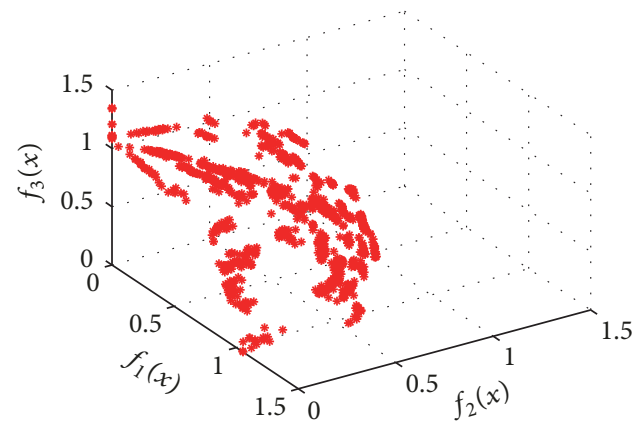

(e) NSGA-II-TS (100 variables)

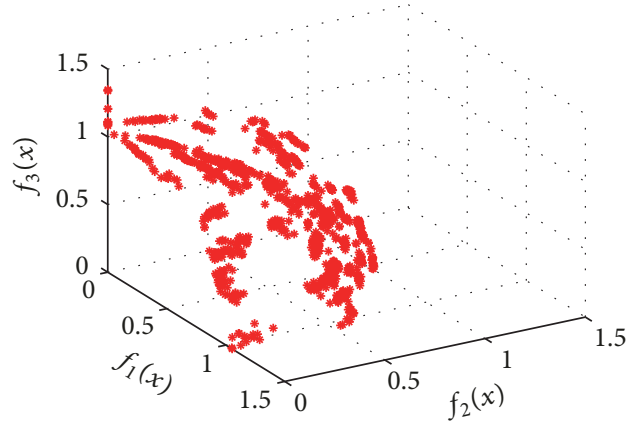

(b) NSGA-II-TS (30 variables)

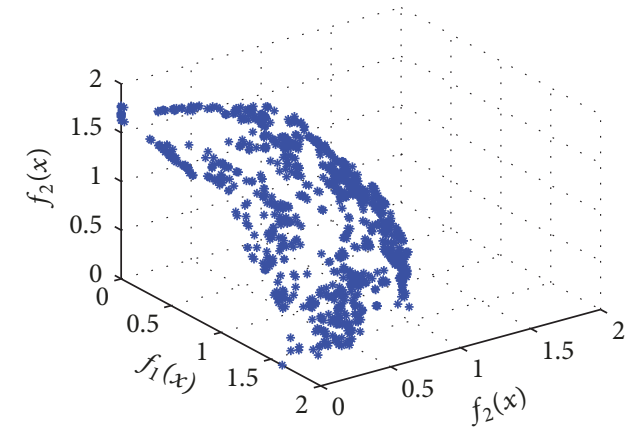

(d) NSGA-II (100 variables)

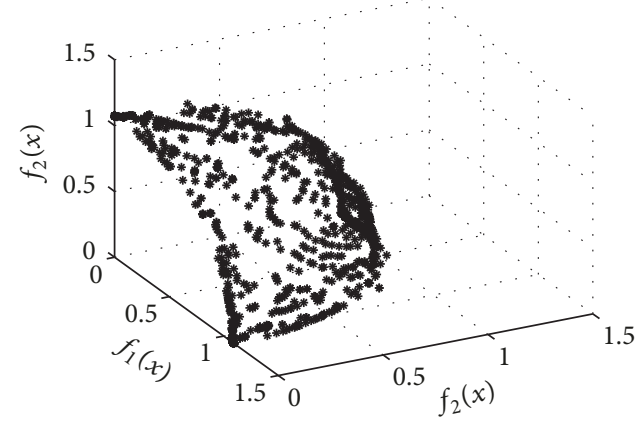

(f) MOEA/D (100 variables)

Figure 2: Pareto front for DTLZ2. 


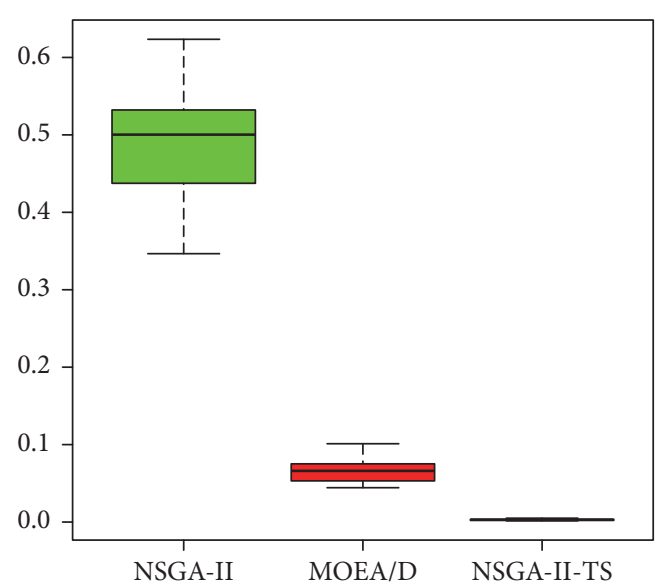

(a) ZDT1-30-gd

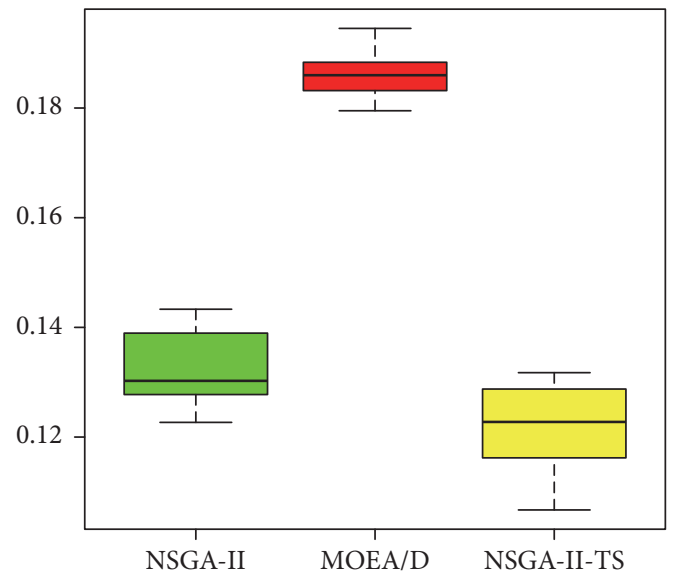

(c) DTLZ2-30-gd

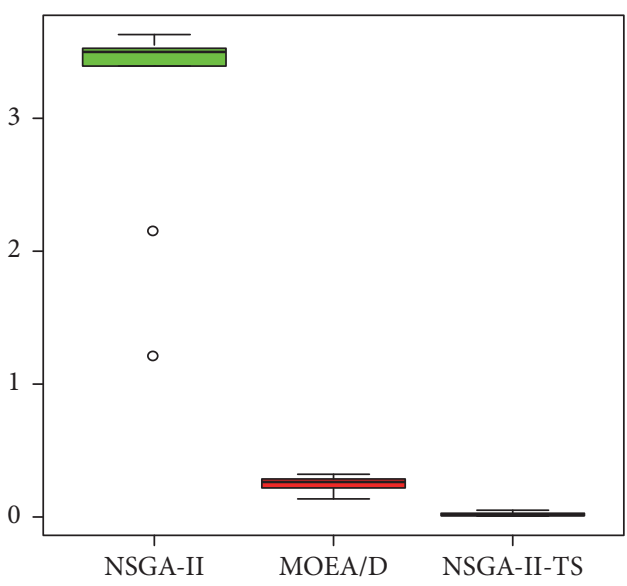

(e) ZDT 2-100-gd

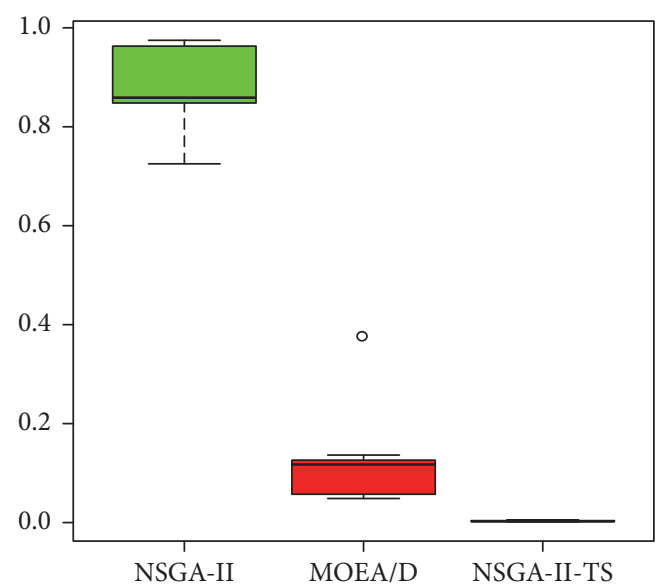

(b) ZDT 2-30-gd

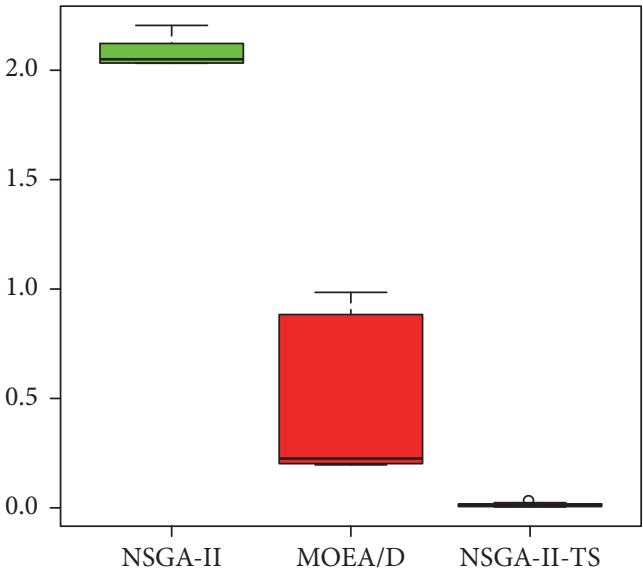

(d) ZDT 1-100-gd

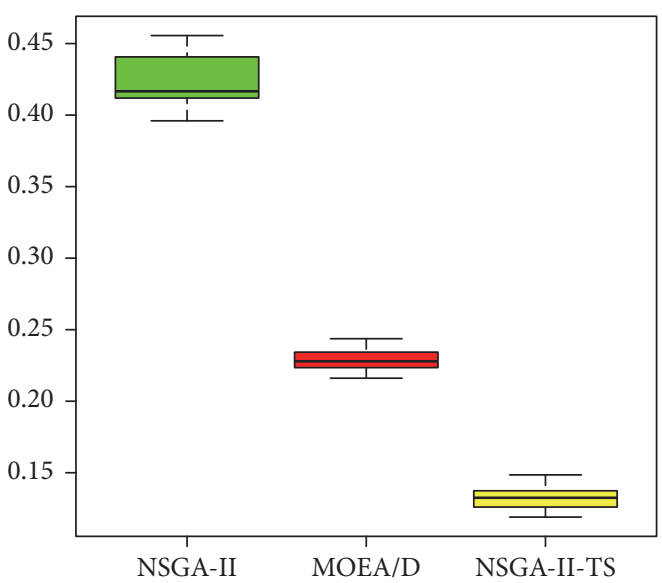

(f) DTLZ2-100-gd

FIGURE 3: The box chart of generation distance for test functions.

$A$ and $B$ of the optimization problem. The calculation is as follows:

$$
\mathrm{sp}=\sqrt{\frac{1}{n-1} \sum_{i=1}^{n}\left(\bar{d}-d_{i}\right)^{2}}
$$

where $d_{i}$ refers to the distance between each solution of the nondominated set and the nearest neighbor of its, $\bar{d}$ is the mean value of $d_{i}$, and $n$ is the number of solutions in nondominated set; the smaller the sp index value, the better the distribution of the nondominated set.

Figure 4 presents the box plot of SP obtained by NSGAII-TS, NSGA-II, and MOEA/D in the case of ZDT1, ZDT2, 


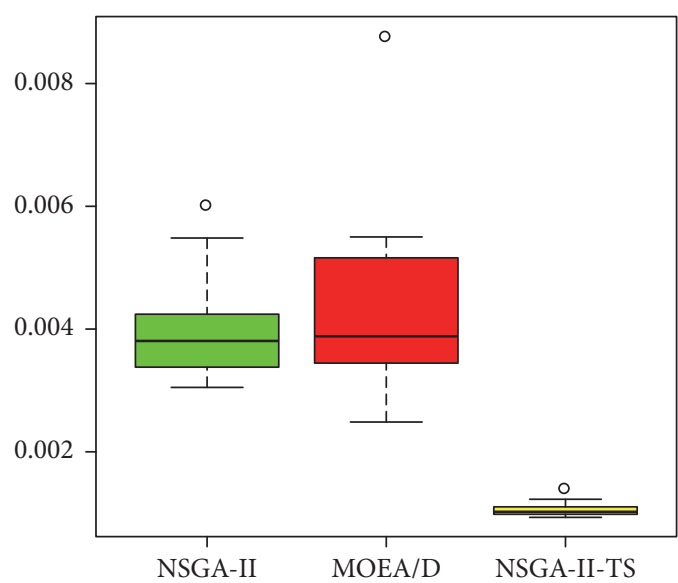

(a) ZDT1-30-sp

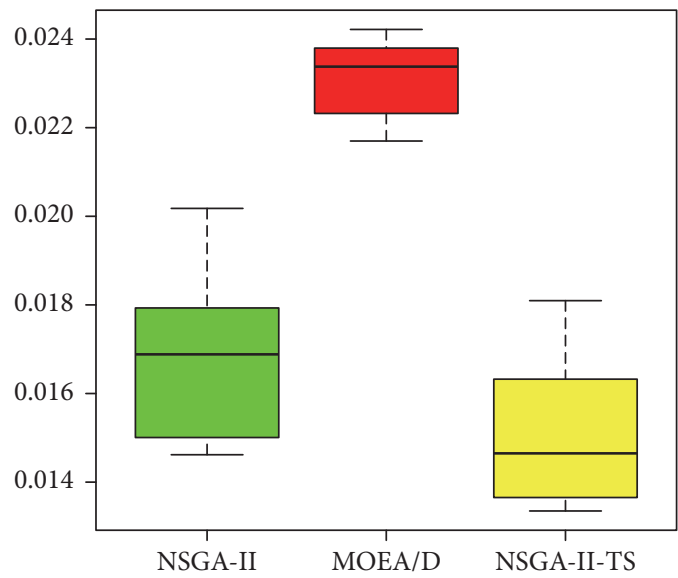

(c) DTLZ2-30-sp

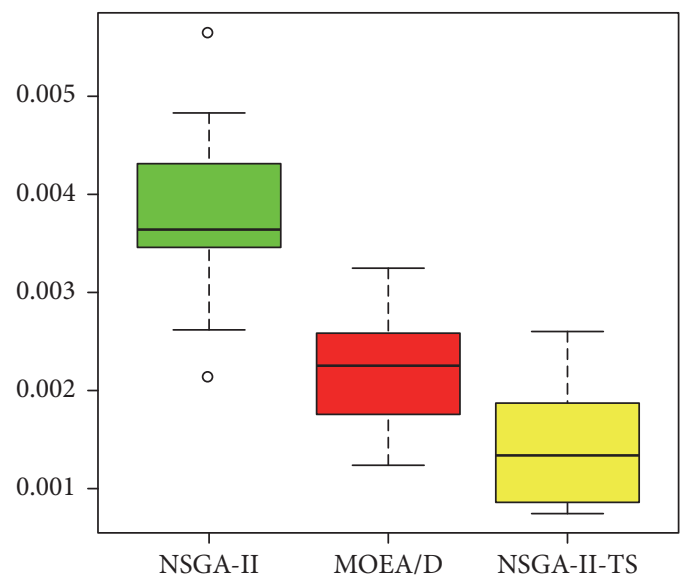

(e) ZDT 2-100-sp

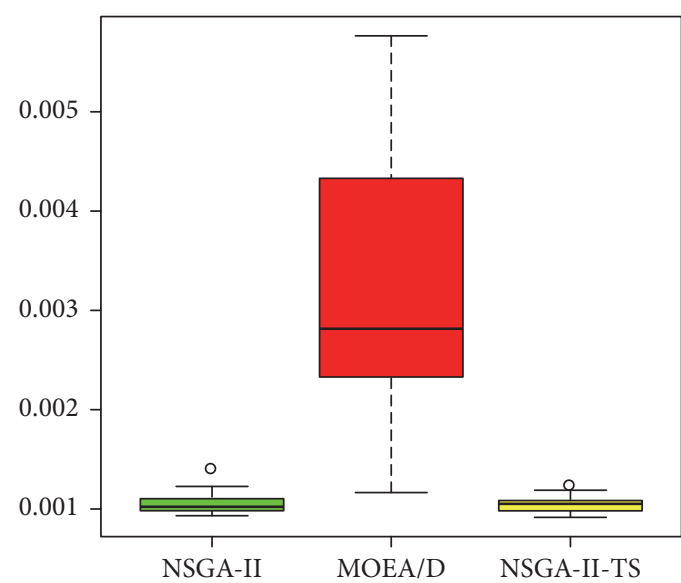

(b) ZDT 2-30-sp

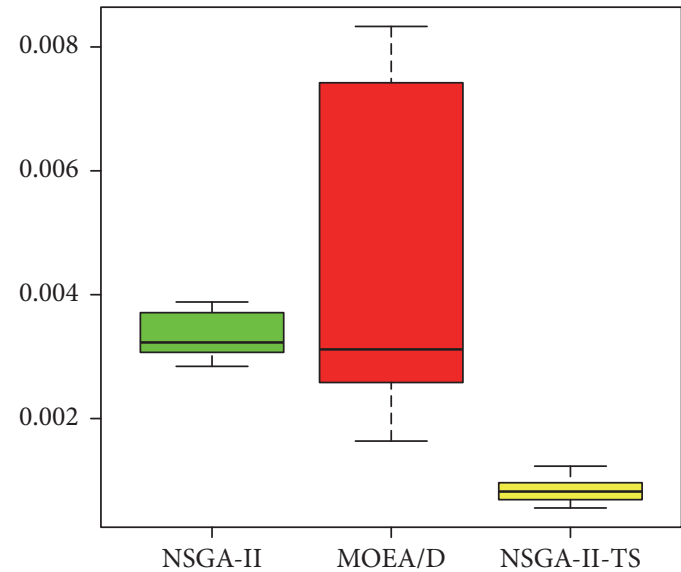

(d) ZDT 1-100-sp

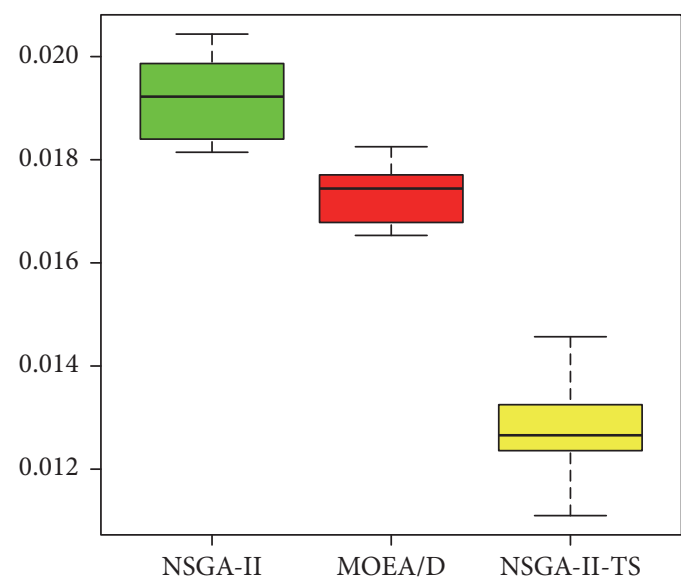

(f) DTLZ2-100-sp

Figure 4: The box chart of sp for test functions.

and DTLZ2 with different variables, which illustrates the dispersion of SP. On the whole, we can see that the SP of NSGAII, MOEA/D, and NSGA-II-TS are small, which means the distributions of the three algorithms are pretty good, but from individual, we can observe that the proposed NSGA-II-TS algorithm has the minimum SP, and its deviation is smallest, which means, compared with NSGA-II and MOEA/D, the Pareto front's distribution of the NSGA-II-TS is optimal, and the property still exist when the number of independent variables increases to 100 .

Table 3 shows the mean, standard deviation, and median value of the SP for NSGA-II-TS, NSGA-II, and MOEA/D. 
TABLE 3: SP.

\begin{tabular}{|c|c|c|c|c|c|c|c|c|c|}
\hline & \multicolumn{3}{|c|}{ TS } & \multicolumn{3}{|c|}{ NSGA-II } & \multicolumn{3}{|c|}{ MOEA/D } \\
\hline & Mean & Std. dev. & Median & Mean & Std. dev. & Median & Mean & Std. dev. & Median \\
\hline \multicolumn{10}{|l|}{ ZDT1 } \\
\hline 30 & 0.0011 & 0.0001 & 0.0010 & 0.0041 & 0.0009 & 0.0038 & 0.0044 & 0.0017 & 0.0038 \\
\hline 100 & 0.0008 & 0.0002 & 0.0008 & 0.0033 & 0.0003 & 0.0032 & 0.0043 & 0.0024 & 0.0031 \\
\hline \multicolumn{10}{|l|}{ ZDT2 } \\
\hline 30 & 0.0011 & 0.0009 & 0.0024 & 0.0043 & 0.0018 & 0.0039 & 0.0031 & 0.0013 & 0.0028 \\
\hline 100 & 0.0014 & 0.0006 & 0.0013 & 0.0009 & 0.7632 & 0.0036 & 0.0023 & 0.0006 & 0.0022 \\
\hline \multicolumn{10}{|c|}{ DTLZ2 } \\
\hline 12 & 0.0150 & 0.0015 & 0.0146 & 0.0167 & 0.0017 & 0.0169 & 0.0231 & 0.0008 & 0.0233 \\
\hline 100 & 0.0129 & 0.0014 & 0.012 & 0.0191 & 0.0007 & 0.0192 & 0.0173 & 0.0006 & 0.0174 \\
\hline
\end{tabular}

TABLE 4: The average value of objectives.

\begin{tabular}{lcccccc}
\hline & \multicolumn{2}{c}{${\text { Utility } / \mathrm{m}^{3}}$} & \multicolumn{2}{c}{ Facilities/pieces } & \multicolumn{2}{c}{ Satisfaction/\% } \\
& Mean & Std. dev. & Mean & Std. dev. & Mean & 0.911 \\
NSGA-II & 46440 & 893 & 16 & 2.5 & 0.045 \\
NSGA-II-TS & 47258 & 90 & 18 & 1.5 & 0.957 & 0.002 \\
MOEA/D & 47143 & 113 & 45 & 2.4 & 0.951 & 0.008 \\
\hline
\end{tabular}

From Table 3, the mean value of NSGA-II-TS on ZDT1 is 0.0011 , the standard deviation is 0.0001 , the mean value of NAGA-II is 0.0041 , the standard deviation is 0.0009 , the mean value of MOEA/D is 0.0044 , and the standard deviation is 0.0017. The experimental results show that the distribution of NSGA-II-TS is optimal, and its distribution did not sharply change with the increase of the number of variables, which illustrates that the distribution of NSGA-II-TS is higher than NSGA-II and MOEA/D from the numerical description.

The results of the comparison experiments show that both of the convergence and the distribution of Pareto front of NSGA-II-TS are much better than NSGA-II and MOEA/D through the three indexes which are Pareto front, generation distance, and spacing. The introduction of the tabu search algorithm in elitism of NSGA-II can avoid falling into local optimum and keep the diversity among the obtained Pareto optimal solutions. With the number of the variables increasing, the advantage is obvious. In summary, the proposed NSGA-II-TS algorithm has good diversity and convergence in solving multiobjective functions of large-scale variables.

\section{Application Analyses}

We set the number of candidates facilities to 100, the fixed facilities to 3 , and the demand points to 10, and the algorithm is performed for 100 iterations with a population number of 100 to achieve the Pareto front, being independently run 20 times. The nondominated set is obtained by NSGA-II, MOEA/D, and NSGA-II-TS, respectively. But the criterion of the combined solution superiority is not given. Therefore, this paper adopts the ideal point method [14] to evaluate the extent of the combination of solutions.

Due to the fact that the incommensurateness between the objective functions should be solved, in this paper the $z$ score normalization is used to normalize the three objective functions. For solution $h$, the normalized objective function $f_{i}^{*}(h)$ is given as

$$
f_{i}^{*}(h)=\frac{f_{i}(h)-\bar{f}_{i}}{\sigma\left(f_{i}\right)}, \quad i=1,2,3, h \in S
$$

where $\bar{f}_{i}$ denotes the mean value of $f_{i}$ for solutions in $S$ and $\sigma\left(f_{i}\right)$ denotes the standard deviation of $f_{i}$ for solutions in $S$.

Second, the ideal point is defined as

$$
\begin{aligned}
F^{*} & =\left\{f_{1}^{\text {ideal }}, f_{2}^{\text {ideal }}, f_{3}^{\text {ideal }}\right\} \\
& =\left\{\max \left\{f_{1}{ }^{*}\right\}, \min \left\{f_{2}{ }^{*}\right\}, \min \left\{f_{3}{ }^{*}\right\}\right\} .
\end{aligned}
$$

Then the Euclidean distance $d(h)$ from each $h$ to the ideal point can be calculated as

$$
d(h)=\sqrt{\sum_{i}\left(f^{*}{ }_{i}-f_{i}^{\text {ideal }}\right)^{2}} .
$$

Finally, the solutions with the minimum value of $d(h)$ are selected as the best compromise solutions.

Table 4 shows the optimal solution obtained by two-stage method for each algorithm. It can be seen from Table 4 that NSGA-II is able to yield better optimal solutions as compared to NSGA-II and MOEA/D. From Table 4, we can see, for the objective of effectiveness, the value of traditional NSGA-II is less than the modified one for 818 cubic and the value of MOEA/D is less than the modified one for 115 cubic which is large enough. For the second objective, the value of the proposed NSGA-II-TS algorithm is larger than NSGA-II for the other two, but it is half the value of the MOEA/D algorithm. For the third objective, the value yielded by NSGA-II-TS algorithm is marginally better than NSGA-II and MOEA/D. Thus, compared with NSGA-II, the proposed 

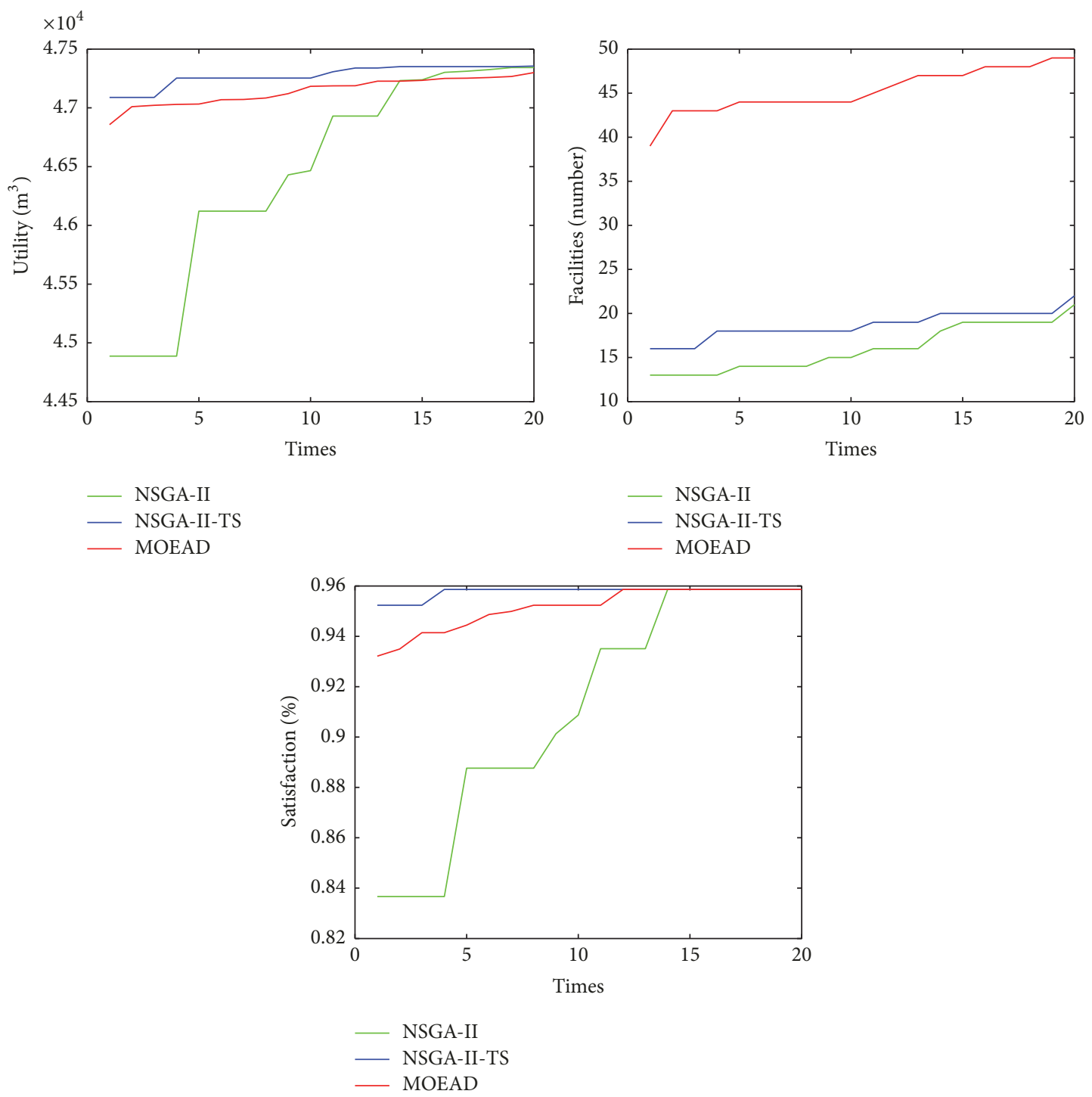

FIGURE 5: Analyses of different objectives.

NSGA-II-TS algorithm, on the basis of high effectiveness and the satisfaction of the disaster areas, it is worth increasing two emergency facilities. Compared with MOEA/D, as the levels of effectiveness and satisfaction of the disaster areas are comparable, the number of temporary facilities is half that of MOEA/D which has less cost. Above all, we can conclude that the performance of the proposed NSGA-II-TS algorithm is better than the traditional methods.

Figure 5 shows the comparative analysis of the optimal solution obtained by NSGA-II, MOEA/D, and NSGA-IITS based on the two-stage metric. As can be seen from Figure 5, the modified NSGA-II surpasses the traditional one according to two-stage metric in all experimental problems. For first objective, the highest deviation of traditional NSGAII from the modified one is $24.21 \%$ for problems of size 100 , and the average deviation is $9.58 \%$. For the second objective, the corresponding highest deviation is $2.29 \%$ for problems of size 100 , and the average is $0.5 \%$. For the third objective, the corresponding highest deviation is $2.29 \%$ for problems of size 100 , and the average is $0.5 \%$. Combining the above data with those in Table 3, we can conclude that the performance of the proposed NSGA-II-TS algorithm is better than the traditional multiobjective algorithm MOEA/D and NSGA-II.

Table 5 shows the difference degree analysis of NSGAII, MOEA/D, and NSGA-II-TS based on rank sum test with Kruskal-Wallis. The results show that the significant levels of three algorithms were $0.1,0.0$, and 0.024 in terms of material utility, number of temporary facilities, and satisfaction, which rejects the original hypothesis: material utility, the number of temporary facilities, and satisfaction in different algorithm are related; namely, the results of the three algorithms have significant difference statistically, which supports the analysis 
TABLE 5: Hypothesis test.

\begin{tabular}{|c|c|c|c|c|}
\hline Number & Original hypothesis & Test & Significance level & Decision maker \\
\hline (1) & $\begin{array}{l}\text { The distribution of the material } \\
\text { utility is the same as the } \\
\text { algorithm }\end{array}$ & Kruskal-Wallis & .010 & Deny \\
\hline (2) & $\begin{array}{l}\text { The distribution of the number } \\
\text { of temporary facilities is the same } \\
\text { as the algorithm }\end{array}$ & Kruskal-Wallis & .000 & Deny \\
\hline (3) & $\begin{array}{c}\text { The distribution of satisfaction in } \\
\text { disaster area is the same as the } \\
\text { algorithm }\end{array}$ & Kruskal-Wallis & .024 & Deny \\
\hline
\end{tabular}

TABLE 6: Distribution scheme.

\begin{tabular}{|c|c|c|}
\hline Number & Facilities & Quantity $\left(\mathrm{m}^{3}\right)$ \\
\hline \multirow{2}{*}{ (1) } & 4 & 2915 \\
\hline & 16 & 1585 \\
\hline \multirow{3}{*}{ (2) } & 2 & 500 \\
\hline & 5 & 1300 \\
\hline & 9 & 3500 \\
\hline \multirow{2}{*}{ (3) } & 8 & 1200 \\
\hline & 18 & 3000 \\
\hline \multirow{4}{*}{ (4) } & 1 & 400 \\
\hline & 4 & 85 \\
\hline & 11 & 3200 \\
\hline & 17 & 1315 \\
\hline \multirow{2}{*}{ (5) } & 7 & 500 \\
\hline & 13 & 4000 \\
\hline$(6)$ & 8 & 3000 \\
\hline$(7)$ & 17 & 3500 \\
\hline \multirow{3}{*}{ (8) } & 3 & 800 \\
\hline & 6 & 4000 \\
\hline & 12 & 2200 \\
\hline (9) & 15 & 5500 \\
\hline \multirow{2}{*}{ (10) } & 10 & 2500 \\
\hline & 14 & 3500 \\
\hline
\end{tabular}

based on Table 3 and verifies that the performance of NSGAII-TS is superior to traditional multiobjective algorithms NSGA-II and MOEA/D again.

Table 6 shows that the distribution scheme for the number of temporary facilities is 18 . As can be seen from Table 6, the selected facilities can meet the requirements of the demand points without waste.

From the analyses of Tables 4-6 and Figure 5, we can clearly find out that NSGA-II-TS is more effective in dealing with large-scale emergency location problem. It can be seen that the improvement of this algorithm is effective, and with the increase of scale of emergency location, the superiority of the algorithm is more obvious.

\section{Conclusions}

This paper proposes a dynamic multiobjective location model for large-scale emergencies management with three factors being considered. The model proposed in this paper enhances the robustness of the emergency management by considering the fact that the advanced emergency centers may no longer be sufficient to deal with the needs of the affected areas in a serve crisis that affects a large area.

We provide a practical optimization method to select the locations of emergency response facilities in the stage of rescuing. The method proposed a hybrid elitism merging the parent individuals, genetic operator individuals, and tabu search operator individuals, thus reducing the duplicate individuals in the process of search and improving the diversity of the offspring individuals.

The computational experiments are divided into two sections: firstly the numerical experiment is constructed by the classical functions ZDT1, ZDT2, and DTLZ2, through the Pareto front, generation distance, and spacing to verify that both the convergence and uniformity of the proposed algorithm are better than those of the NSGA-II and MOEA/D, providing a general approach for the multiobjective optimization design of the emergency facilities location. Then, we use a case study on relief goods for earthquakes at disaster response facilities, using ideal point to set a criterion to evaluate the superiority of combinatorial solutions. The results obtained by using the proposed method are compared with those obtained by NSGA-II and MOEA/D. The comparison results show that the proposed method yields better results in lower facilities and higher material utility and satisfaction. The algorithm proposed in this paper has high application value in the emergency management of sudden disaster crisis and other security system construction.

\section{Conflicts of Interest}

The authors declare that they have no conflicts of interest.

\section{Acknowledgments}

This research is supported by the National Natural Science Foundation of China (61573012), the Opening Project of Hubei Collaborative Innovation Center for Early Warning and Emergency Response Technology (no. JD20160214), Fundamental Research Funds for the Central University (WUT: 2016A004), Fundamental Research Funds for the Central University (2017-zy-116), and the Key Cultivation Projects in Wuhan University of Technology (471-39101075). 


\section{References}

[1] F. S. Salman and E. Yücel, "Emergency facility location under random network damage: Insights from the Istanbul case," Computers \& Operations Research, vol. 62, pp. 266-281, 2015.

[2] R. Abounacer, M. Rekik, and J. Renaud, "An exact solution approach for multi-objective location-transportation problem for disaster response," Computers \& Operations Research, vol. 41, no. 1, pp. 83-93, 2014.

[3] R. H. Ballou, "Dynamic warehouse location analysis," Journal of Marketing Research, vol. 5, no. 3, p. 271, 1968.

[4] S. Gao, Y. Wang, J. Cheng, Y. Inazumi, and Z. Tang, "Ant colony optimization with clustering for solving the dynamic location routing problem," Applied Mathematics and Computation, vol. 285, pp. 149-173, 2016.

[5] M. Marufuzzaman, R. Gedik, and M. S. Roni, "A Benders based rolling horizon algorithm for a dynamic facility location problem," Computers \& Industrial Engineering, vol. 98, pp. 462469, 2016.

[6] K. R. Gue, "A dynamic distribution model for combat logistics," Computers \& Operations Research, vol. 30, no. 3, pp. 367-381, 2003.

[7] R. Manzini and E. Gebennini, "Optimization models for the dynamic facility location and allocation problem," International Journal of Production Research, vol. 46, no. 8, pp. 2061-2086, 2008.

[8] H. Jia, F. Ordóñez, and M. Dessouky, "A modeling framework for facility location of medical services for large-scale emergencies," Institute of Industrial Engineers (IIE). IIE Transactions, vol. 39, no. 1, pp. 41-55, 2007.

[9] L. Baken, B. Benoit, A. H. J. Koning, P. J. Van Der Spek, E. A. P. Steegers, and N. Exalto, "First-Trimester Crown-Rump Length and Embryonic Volume of Fetuses with Structural Congenital Abnormalities Measured in Virtual Reality: An Observational Study," BioMed Research International, vol. 2017, Article ID 1953076, 2017.

[10] Y. Deng, W. Zhu, J. Tang, and J. Qin, “Solving a two-stage stochastic capacitated location-allocation problem with an improved PSO in emergency logistics," Mathematical Problems in Engineering, Art. ID 6710929, 15 pages, 2017.

[11] C. Wang, Z. Ji, and Y. Wang, "A Novel Memetic Algorithm Based on Decomposition for MULtiobjective Flexible Job Shop SCHeduling Problem," Mathematical Problems in Engineering, Art. ID 2857564, 20 pages, 2017.

[12] X. Li and Y. Fang, "Dynamic Environmental/Economic Scheduling for Microgrid Using Improved MOEA/D-M2M," Mathematical Problems in Engineering, vol. 2016, Article ID 2167153, 2016.

[13] B. Gadhvi, V. Savsani, and V. Patel, "Multi-Objective Optimization of Vehicle Passive Suspension System Using NSGA-II, SPEA2 and PESA-II," Procedia Technology, vol. 23, pp. 361-368, 2016.

[14] A.-D. Li, Z. He, and Y. Zhang, "Bi-objective variable selection for key quality characteristics selection based on a modified NSGA-II and the ideal point method," Computers in Industry, vol. 82, pp. 95-103, 2016.

[15] A. E. Zade, A. Sadegheih, and M. M. Lotfi, "A modified NSGAII solution for a new multi-objective hub maximal covering problem under uncertain shipments," Journal of Industrial Engineering International, vol. 10, no. 4, pp. 185-197, 2014.
[16] L. Wang, T.-G. Wang, and Y. Luo, "Improved non-dominated sorting genetic algorithm (NSGA)-II in multi-objective optimization studies of wind turbine blades," Applied Mathematics and Mechanics-English Edition, vol. 32, no. 6, pp. 739-748, 2011.

[17] J. Dong, Q. Li, and L. Deng, "Fast multi-objective optimization of multi-parameter antenna structures based on improved MOEA/D with surrogate-assisted model," AEÜ - International Journal of Electronics and Communications, vol. 72, pp. 192-199, 2017.

[18] L. Cai and Y. Sheng, "Hom-Big Brackets: Theory and Applications," Symmetry, Integrability and Geometry: Methods and Applications, 2016.

[19] T. Jiang, "A tabu search approach to optimal structuring element extraction for MST-based shapes description," International Journal of Computer Mathematics, vol. 71, no. 4, pp. 437-445, 1999.

[20] X. Zhang, S. Zhong, Y. Liu, and X. Wang, "A framing link based Tabu search algorithm for large-scale multi-depot vehicle routing problems," Mathematical Problems in Engineering, vol. 2014, Article ID 152494, 13 pages, 2014.

[21] F. Y. Qiu, L. P. Mo, B. Jiang, and L. P. Wang, "Multi-objective particle swarm optimization algorithm using large scale variable decomposition," Chinese Journal of Computers. Jisuanji Xuebao, vol. 39, no. 12, pp. 2598-2613, 2016.

[22] K. Deb, A. Pratap, S. Agarwal, and T. Meyarivan, "A fast and elitist multiobjective genetic algorithm: NSGA-II," IEEE Transactions on Evolutionary Computation, vol. 6, no. 2, pp. 182-197, 2002.

[23] J. R. Schott, "Fault tolerant design using single and multicriteria genetic algorithm optimization," Cellular Immunology, vol. 37, no. 1, pp. 1-13, 1995. 


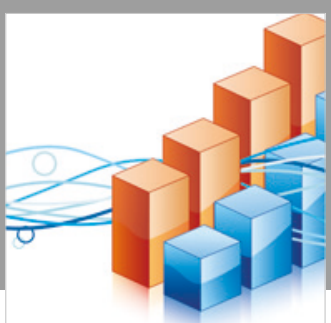

Advances in

Operations Research

\section{-n-m}
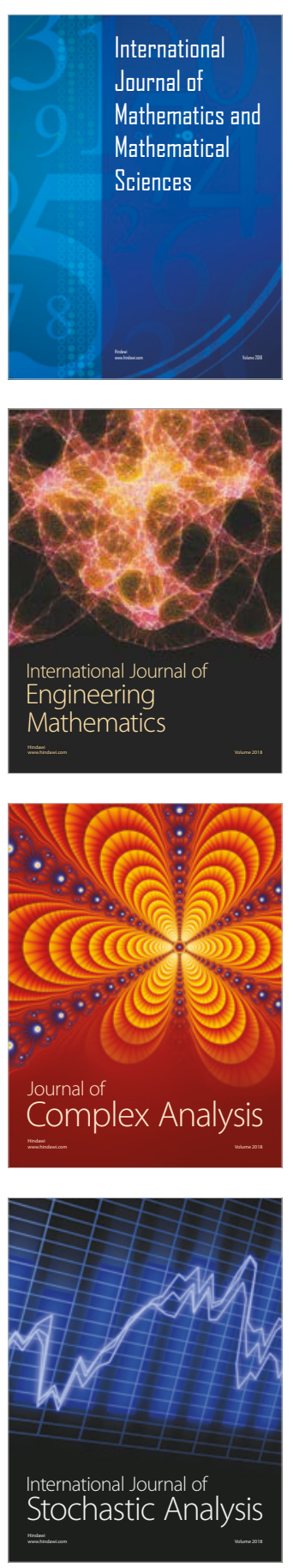
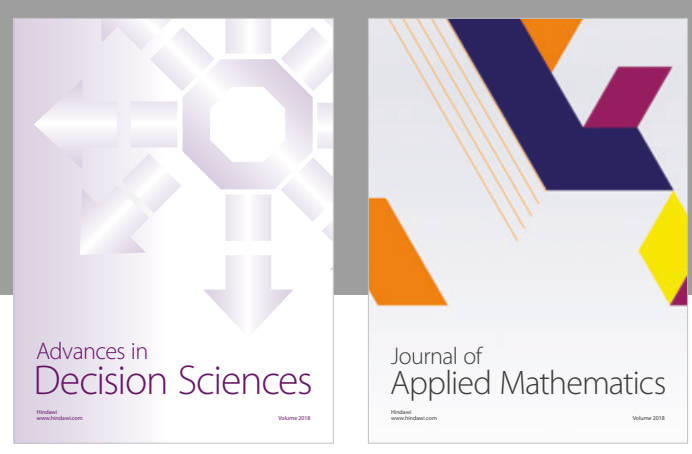

Journal of

Applied Mathematics
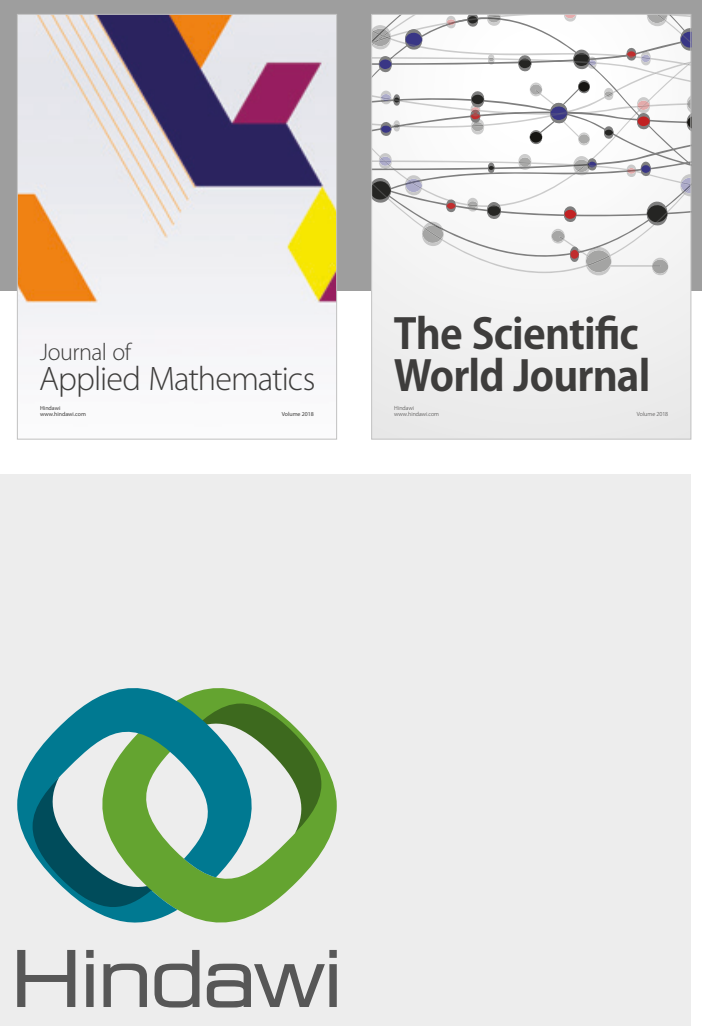

Submit your manuscripts at

www.hindawi.com

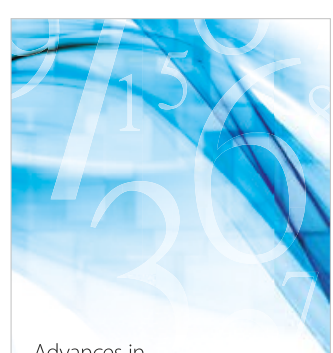

Advances in
Numerical Analysis
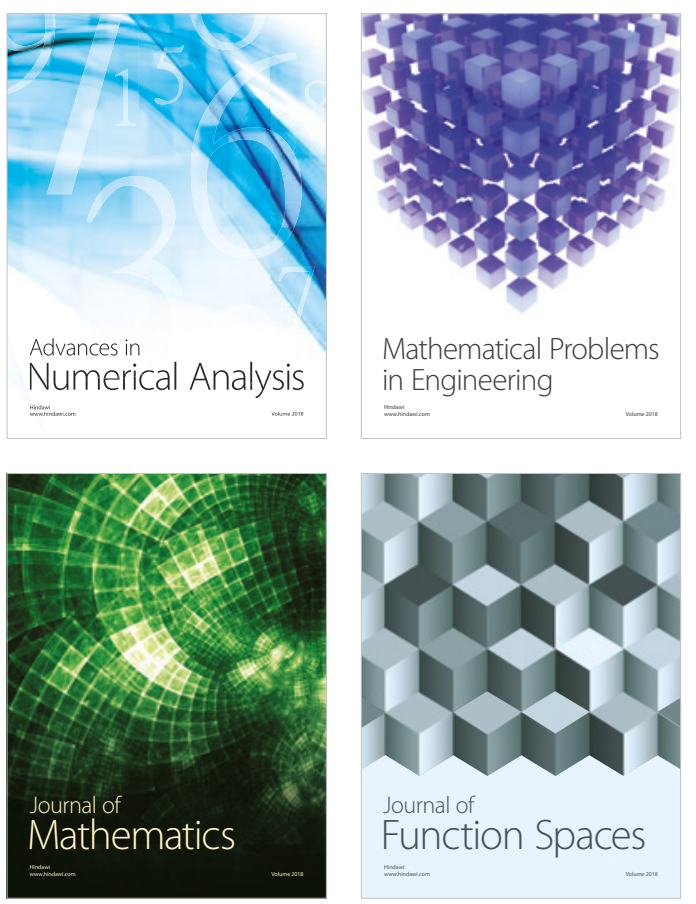

Mathematical Problems in Engineering

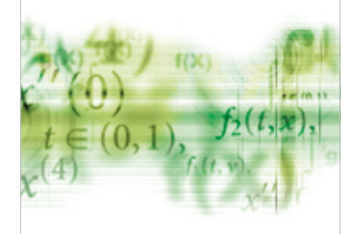

International Journal of

Differential Equations

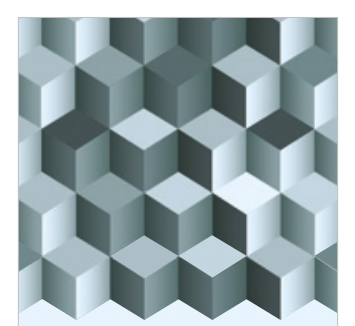

Journal of

Function Spaces

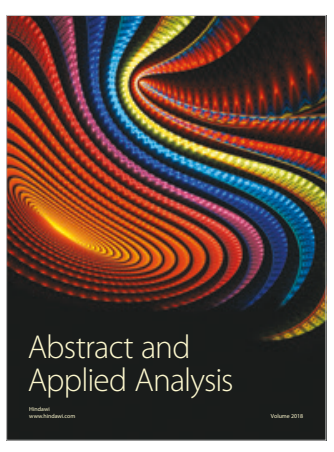

The Scientific

World Journal

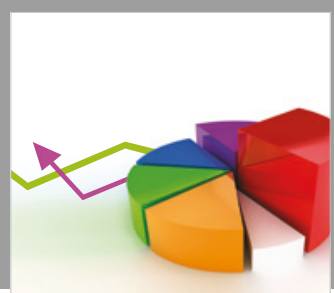

Journal of

Probability and Statistics
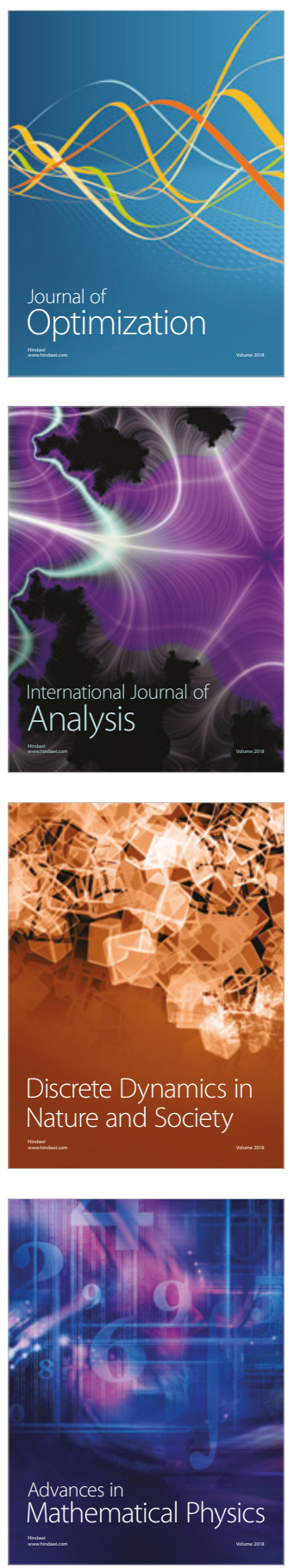